\title{
Uma nova abordagem para a segmentação de pulmões utilizando o método de contorno ativo não paramétrico Optimum Path Snakes em imagens de tomografia computadorizada
}

\author{
Aldísio G. Medeiros ${ }^{1}$, Solon A. Peixoto ${ }^{1}$, Antonio Carlos S. Barros ${ }^{2}$, \\ Victor Hugo C. de Albuquerque ${ }^{2}$ e Pedro P. Rebouças Filho ${ }^{1}$ \\ ${ }^{1}$ Instituto Federal de Ciência e Tecnologia do Ceará \\ - Fortaleza - CE - Brasil \\ ${ }^{2}$ Universidade de Fortaleza - Fortaleza - CE - Brasil \\ \{aldisio.medeiros, solon.alves, carlosbarros\}@lapisco.ifce.edu.br \\ victor.albuquerquedunifor.br, pedrosarfeifce.edu.br
}

\begin{abstract}
This work propose a new approach to segmentation of lungs on computed tomography images using an adaptative method based on active contour called Optimum Path Snakes (OPS). This method is combined with Analysis of Human Tissue Densities (AHTD), a new method for feature extraction. The analysis examined 72 lung images of humans volunteer with fibrosis, chronic obstructive pulmonary disease and healthy. The results shows that OPS method combined with AHTD extractor has competitive potencial when compared to other segmentation methods, reaching values of Accuracy and Sensitivity in the order of 99,88\% $\pm 00,12$ and 99,25\% $\pm 00,33$, respectively. With degree of similarity above $95 \%$ when compared to ground thru, on average. We can conclude that the proposed approach can be used as an aid for medical diagnosis in pulmonology.
\end{abstract}

Resumo. Este trabalho propõe uma nova abordagem para a segmentação pulmonar utilizando o método adaptativo e não paramétrico baseado em contornos ativos chamado Optimum Path Snakes (OPS), em imagem de tomografia computadorizada. O método é combinado com o extrator Análise de Densidades dos Tecidos Humanos (ADH) para a extração de características. Foram utilizadas 72 imagens pulmonares de voluntários com fibrose, doença pulmonar obstrutiva crônica e sadios. Os resultados mostram que o OPS combinado com ADH apresenta potencial competitivo frente aos demais métodos comparados, alcançando valores de Acurácia e Sensibilidade na ordem de 99,88\% $\pm 00,12$ e $99,25 \% \pm 00,33$, respectivamente. Com índices de similaridade ao padrão-ouro superiores a 95\%, em média, podemos concluir que a abordagem proposta pode ser utilizada para auxílio ao diagnóstico médico em pneumologia.

\section{Introdução}

As patologias pulmonares vêm ocupando posições preocupantes dentre as doenças que mais causam mortes em todo o mundo. Somente no ano de 2015 houve um aumento de 
$30 \%$ no número de mortes por doença pulmonar obstrutiva crônica (DPOC), patologia que em 2002 ocupava a quinta posição dentre as maiores taxas de mortalidade. Segundo da Silva Barros [2016], já estima-se que até 2030 se torne a terceira maior causa de morte no mundo em decorrência do desenvolvimento urbano e da baixa qualidade de vida.

Segundo Pauwels et al. [2012], a DPOC caracteriza-se como uma redução progressiva no fluxo respiratório que pode ser causada por bronquite crônica ou enfisema, obstruindo o espaço aéreo pulmonar e reduzindo a capacidade de oxigenação. Deste modo, o diagóstico precoce dedica-se a detecção do grau de aeração pulmonar, sendo a tomografia computadorizada (TC) o exame de escolha na avaliação diagnóstica de doenças no parênquima do pulmão [da Silva Felix et al., 2009]. Neste contexto, o auxílio ao diagnóstico médico tem-se mostrado uma importante ferramenta clínica, que além de contribuir para a detecção de artefatos oferece aos especialistas uma segunda opinião.

Considerando os sistemas de apoio ao diagnóstico baseado em visão computacional, a etapa de segmentação apresenta-se como um passo fundamental para a detecção de indícios. Dentre as técnicas utilizadas em imagens médicas, pode-se citar a aplicação de morfologia matemática [Medeiros and Ferreira, 2015], combinação do crescimento de região com limiares locais [da Silva Felix et al., 2009], além da aplicação da transformada watershed para a segmentação pulmonar [Avinash et al., 2016]. Tais técnicas tradicionais, entretando, não costumam apresentar resultados consistentes para imagens de TC do tórax, segundo Rebouças Filho et al. [2014] isso se deve ao fato das estruturas internas, como artérias, veias e vias aéreas atuarem como ruído, distorcendo o processo de segmentação e comprometendo resultados. Dessa forma, visando contornar estas limitações, destacam-se técnicas baseadas nos métodos de contornos ativos (MCA).

Diante deste cenário, este trabalho propõe uma nova abordagem para a segmentação de pulmões em imagens de TC do tórax utilizando o método adaptativo e não paramétrico baseado em contornos ativos chamado Optimum Path Snakes (OPS). Além disso um novo extrator chamado Análise de Densidades dos Tecidos Humanos (ADH) é utilizado para a representação de características na imagem.

Uma análise é realizada a partir do padrão-ouro (ground truth) construído por um médico especialista. A partir dos resultados é feita uma uma avaliação estatística entre o OPS e outros métodos de segmentação considerados na literatura, além de comparar com o Sistema para a Detecção e a Quantificação de Enfisema Pulmonar (SISDEP), proposto por da Silva Felix et al. [2009]. Para o estudo foram utilizadas imagens de pulmões classificadas com fibrose, DPOC e sadias.

\section{Fundamentação Teórica}

Métodos tradicionais podem apresentar limitações quando aplicados a segmentação de pulmões em imagens de TC do tórax, segundo Rebouças Filho et al. [2014], isso acontece por conta das imprecisões geradas pelas estruturas internas do parênquima pulmonar. Afim de contornar essas limitações, as técnicas de contornos ativos baseiam-se em métodos que visam a minimização de uma função que representa a deformação da curva, que evolui no sentido de compreender totalmente a região de interesse.

Inicialmente o método baseado em contornos ativos foi proposto por Kass et al. [1987] e se baseia em modelos deformáveis, onde o objetivo é adaptar uma curva inicial 
ao contorno da região de interesse, esta curva é deformada pela atuação de forças e evolui até as bordas do objeto. A deformação é regida por uma função de energia $E$ a ser minimizada, podendo ser calculada por

$$
E=\sum_{p=0}^{N-1}\left\{E_{\text {int }}[c(p)]+E_{\text {ext }}[c(p)]\right\},
$$

onde $E_{\text {int }}$ representa a energia interna que é relativa às propriedades da região interna da curva de segmentação $c$, em um determinado ponto $c(p)$, e pode ser descrita como

$$
E_{\text {int }}[c(p)]=\alpha c^{\prime}(p)+\beta c^{\prime \prime}(p),
$$

onde os termos $\alpha$ e $\beta$ definem pesos que regem o grau de curvatura, $c^{\prime}(p)$ e $c^{\prime \prime}(p)$ representam a primeira e segunda derivada no ponto $c(p)$ da curva, respectivamente. Já a energia externa, denotada por $E_{\text {ext }}[c(p)]$, depende das propriedades que são externas à curva, tais como linhas, segmentos e terminações. Desta maneira, através de um processo iterativo, a curva $c$ é deformada pela busca de mínimos locais, reduzindo o valor da energia $E$ e assim atraindo ou repelindo o contorno da curva até as bordas do objeto de interesse.

\subsection{Métodos de Segmentação}

Conforme descrevem Rebouças Filho et al. [2014], o MCA Tradicional apresenta limitações na segmentação de regiões com saliências e bifurcações, além de necessitar ser inicializado próximo às bordas do objeto de interesse. Dessa forma, surgem novos MCAs que visam superar tais limitações, alguns propondo novas energias internas, enquanto outros propõem novas energias externas, como é o caso do Gradient Vector Flow (GVF), descrito por $\mathrm{Xu}$ and Prince [1998], inspira-se em vetores de gradiente para adaptação da curva. Embora o GVF solucione o problema de bifurcações, ele é limitado para regiões côncavas onde pode ocorrer a anulação dos vetores de fluxo, assim como também em regiões uniformes, onde o gradiente é nulo ocasionando a estabilização precoce da curva.

Já o MCA Vector Field Convolution (VFC), proposto por Li and Acton [2006], define uma energia externa que pode ser descrita como uma operação de convolução. $\mathrm{O}$ VFC tem como principal vantagem o baixo custo computacional quando comparado aos cálculos de gradiente que são empregados no GVF. Porém, o VFC tem dificuldades quando aplicado em objetos que possuem uma borda de alta intensidade e a outra borda de baixa intensidade, levando a uma segmentação além da região interessada. Para contornar esta limitação da Silva Felix et al. [2013] propuseram o MCA THR modular (THR mod) e o MCA THR multidirecional (THR multi), ambos utilizam a Transformada de Hilbert Radial (THR) 2D para calcular uma nova energia externa, baseando-se na técnica de espalhamento gradual para detecção da borda.

Os métodos citados são inspirados no MCA Tradicional e fazem o uso de uma nova energia externa, entretanto, o cálculo desta energia não faz distinção entre as estruturas internas do parênquima pulmonar que podem atuar como ruídos. Neste sentido, Rebouças Filho et al. [2011] propuseram o MCA CRISP baseado nas faixas de densidade pulmonar, definidas sob unidades Hounsfield cada faixa representa uma classe de densidades. Este método incorpora uma energia externa cujo cálculo é sensível a cada classe de tecido, a partir dos limiares locais esta energia é combinada com a energia interna do 
MCA Balão proposto por Mackiewich [1995]. A limitação deste método está justamente na inicialização da curva, uma vez que o método Balão pode alcançar a estabilidade da curva de forma precoce quando inicializado em regiões descentralizadas e próximo a concavidades [Rebouças Filho et al., 2014].

Como alternativa ao MCA CRISP, Rebouças Filho et al. [2014] propuseram a utilização de uma nova energia balão, além de subtituir a energia externa do CRISP por uma versão adaptativa, compondo assim um novo MCA chamado CRISP Adaptativo (CRAD). Uma de suas vantagens é a utilização de uma Rede Neural Artificial do tipo Multi Layer Perceptron para identificação dos limiares locais considerando as densidades dos tecidos.

\subsection{Extratores de características}

O número de informações em uma imagem pode ser descrito em valores da ordem de $\mathbb{R}^{n}$, onde $n$ é o número de pixels. Logo, é possível perceber que uma análise considerando os pixels em sua totalidade torna-se um trabalho oneroso. Neste sentido, técnicas de extração de características são propostas na literatura como forma de melhor descrever os atributos importantes da imagem usando uma quantidade reduzida de elementos, de forma mais robusta e invariante às mudanças [da Silva Barros, 2016].

Neste contexto estão os momentos espaciais cujos valores escalares são capazes de descrever uma função e capturar suas características, além daqueles utilizados em estatística para a descrição de distribuição de probabilidade, como os momentos estatísticos (MomSta) e invariantes a escala do objeto. Hu [1962] propôs a utilização de sete momentos que são invariantes à escala, rotação e translação, conhecidos como invariantes de Hu (HU). Já a Matriz de Co-ocorrência de Níveis de Cinza (GLCM - Gray Level Co-occurence Matrix) utiliza informações da textura do objeto para estabelecer a dependência espacial dos níveis de cinza de uma imagem, produzindo uma matriz que descreve informações da vizinhança em análise. A partir da GLCM, Haralick et al. [1973] descrevem uma família de medidas estatísticas capazes de representar os atributos espaciais da imagem, tais medidas são conhecidas como descritores de Haralick.

\section{Optimum Path Snakes como uma nova abordagem de segmentação}

Por outro lado, uma nova abordagem de segmentação em imagem de TC do tórax é proposta utilizando o MCA adaptativo de forma não paramétrica e com inicialização automática, denominado Optimum Path Snakes (OPS) [da Silva Barros, 2016].

\subsection{Metodologia}

Segundo da Silva Barros [2016], um ponto $c[p]$ é capaz de analisar a vizinhança e determinar o caminho ótimo para encontrar o objeto de interesse. Por esta razão, cada ponto $p$ da curva $c$ é chamado de explorador. Um ponto explorador é o elemento que sabe onde está e sabe o que está procurando, mas não sabe o que irá encontrar no trajeto de locomoção até o objeto desejado. Dessa forma, a técnica é inspirada no classificador Floresta de Caminhos Ótimos (OPF), descrito por [Papa et al., 2017], e analisa a vizinhança de cada ponto da curva através de uma árvore de menor espalhamento. Cada nó da árvore de caminhos ótimos define uma classe denominada de protótipo que é utilizada para a classificação das amostras, onde o protótipo é sempre o ponto atual do ponto explorador e as amostras são a vizinhança em análise. 
O MCA OPS define uma nova energia total que pode ser representada por

$$
E_{O P S}[c(p)]=E_{\text {intOBA }}[c(p)]+E_{\text {extOP }}[c(p)],
$$

onde o termo $E_{\text {intOBA }}[c(p)]$ descreve uma nova energia interna ótima para determinar o trajeto mais eficaz para que um ponto explorador possa expandir sua curva atual, definindo assim uma força interna denominada balão adaptativa, $F_{O B A}$. Na Figura 1 é apresentada uma curva sob a aplicação da $F_{O B A}$, com a expansão dos pontos $A$ e $F$, a partir do ponto médio dos vizinhos, $M_{O B}$ e $M_{E G}$. Os pontos na região em vermelho, por serem internos a curva, isto é, já explorados, recebem um custo elevado de forma a evitar a exploração em uma região já visitada. Já a energia externa, $E_{\text {extOP }}[c(p)]$, é definida pelo conhecimento que guia cada ponto explorador, este conhecimento é adquirido através do classificador OPF combinado com um extrator de características, é, portanto, descrita como

$$
E_{\text {extOP }}[c(p)]=\left\{\begin{array}{l}
1, \text { quando a amostra (vizinho) está dentro da curva, } \\
F_{O P}[c(p)], \text { caso contrário, }
\end{array}\right.
$$

em que $F_{O P}[c(p)]$ é o custo do protótipo, entre 0 e 1 , obtido no treinamento OPF associado a classe dos objetos não desejados. Percorrendo caminhos ótimos das amostras até os protótipos. Definindo, assim, o quão próximo do ponto explorador está o objeto desejado.

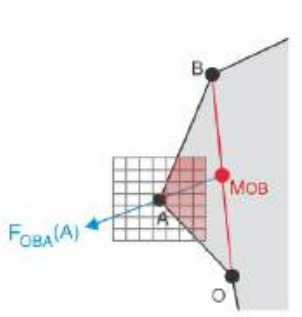

(a)

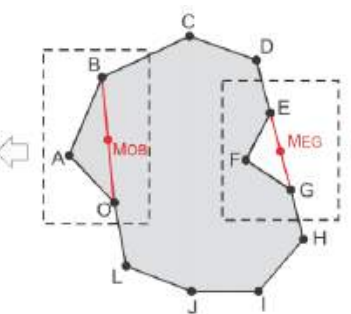

(b)

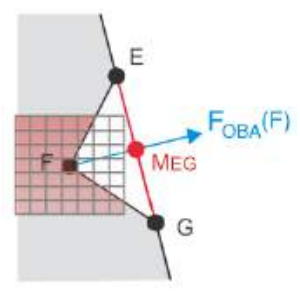

(c)

Figura 1. Exemplo de atuação da força balão adaptativa $F_{O B A}$, com a expansão dos pontos $A$, à esquerda em (a), e $F$, à direita em (b), a partir do ponto médio dos vizinhos, $M_{O B}$ e $M_{E G}$, respectivamente.

\subsection{Análise de Densidades dos Tecidos Humanos (ADH)}

O método de Análise de Densidades dos Tecidos Humanos (ADH) é uma generalização da Análise das Densidades Pulmonares (ADP) baseada em unidades Hounsfield (UH) proposta por Rebouças Filho et al. [2014]. A ADH generaliza esse descritor afim de que possa ser considerado para extração de características em qualquer tipo de imagens médicas, tomando como base o conhecimento das densidades dos tecidos. Os trabalhos de Rebouças Filho et al. [2013, 2014] mostram que é possível identificar padrões entre os pixels de imagens dos pulmões, analisando a composição de sua vizinhança em percentual das faixas radiológicas dos tecidos.

Dessa forma, considerando uma matriz de densidade $D$, a função $f$ determina a quantidade de pontos com densidades presentes em cada classe $u_{i}$ de densidade:

$$
f\left(x, y, u_{i}\right)=\sum_{l=-k}^{k} \sum_{m=-k}^{k} R(x-l, y-m),
$$


com $k=(V-1) / 2$, onde $\mathrm{V}$ é a ordem da vizinhança analisada, e $\mathrm{R}(\mathrm{x}, \mathrm{y})$ pode ser definida como

$$
R(x, y)=\left\{\begin{array}{l}
1, \lim _{\text {inf }}\left(u_{i}\right)<D(x, y)<\lim _{\text {sup }}\left(u_{i}\right), \\
0, \text { caso contrário, }
\end{array}\right.
$$

em que $\lim _{\text {inf }}\left(u_{i}\right)$ e $\lim _{\text {sup }}\left(u_{i}\right)$ representam os limites inferior e superior da faixa de densidade, definida em UH, para a classe $u_{i}$. Assim, ajustando a matriz de densidade $D$ é sugestiva a sua aplicação em outros tipos de tecidos com densidades bem definidas.

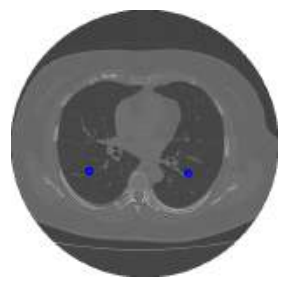

(a)

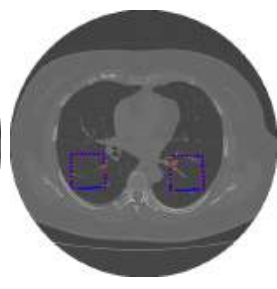

(b)

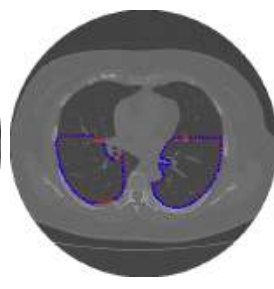

(c)

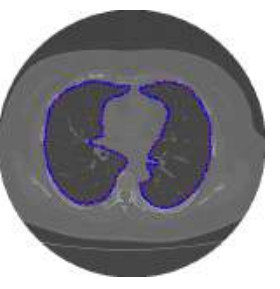

(d)

Figura 2. Exemplo de evolução da curva via OPS, sendo: (a) Curva inicial, (b) 5, (c) 10, (d) 30 iterações (contorno estável).

Na Figura 2 é ilustrada a evolução da curva após iterações de minimização das energias, atingindo neste caso a estabilidade após a trigésima iteração. Na Figura 3 são descritas as etapas do método, desde a abertura da imagem até a estabilização da curva.

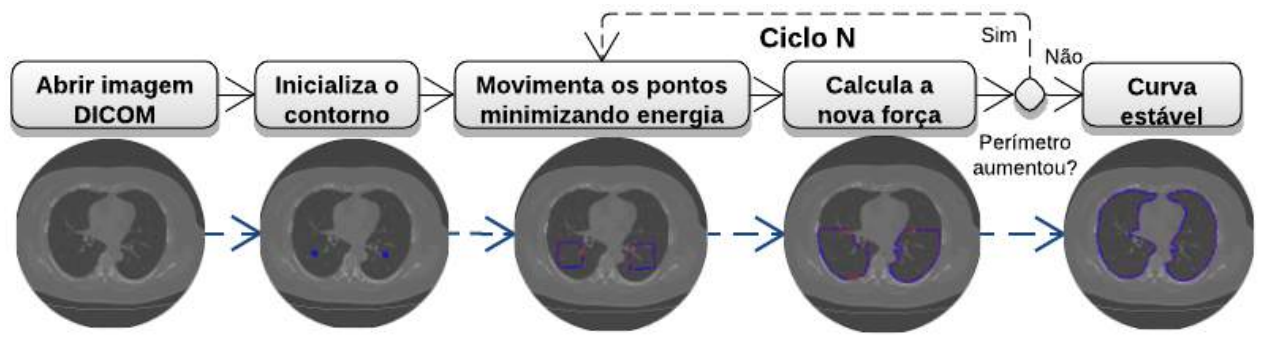

(a)

Figura 3. Diagrama do processo de segmentação do método OPS

\subsection{Base de imagens utilizadas}

As imagens utilizadas para validação do método constituem uma base de imagens obtidas em parceria com o Hospital Walter Cantídio da Universidade Federal do Ceará. Foram cedidas sob a avaliação e aprovação do Comitê de Ética em Pesquisa da UFC - COMEPE (Protocolo $\mathrm{n}^{0}$ 35/06). Os exames foram gerados pelos equipamentos Toshiba Aquilion, GE Medical System LightSpeed16 e Philips Brilliance 10. Todas as imagens são 512x512 pixels com 16 bits e formam uma base de 36 imagens com dois pulmões cada, totalizando um conjunto com 72 imagens de pulmão. O padrão-ouro das imagens analisadas foi obtido por meio da segmentação manual realizada por um pneumologista.

\section{Resultados e Discussões}

A primeira fase da avaliação do método consiste em uma análise do MCA OPS considerando as diferentes combinações de classificadores OPF, bem como extratores de 


\begin{tabular}{|c|c|c|c|c|c|c|}
\hline OPF & Método & Máscara & $\mathrm{Se}$ & Ac & MCC & HD \\
\hline \multirow{4}{*}{ 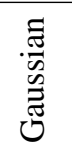 } & $\mathrm{ADH}$ & $15 \times 15$ & $99,88 \pm 00,20$ & $99,25 \pm 00,33$ & $95,05 \pm 01,62$ & $04,02 \pm 00,75$ \\
\hline & MomSta & $15 \times 15$ & $99,05 \pm 02,68$ & $98,67 \pm 02,47$ & $92,32 \pm 12,31$ & $04,73 \pm 01,91$ \\
\hline & $\mathrm{HU}$ & $7 \times 7$ & $99,62 \pm 01,66$ & $99,02 \pm 01,58$ & $92,47 \pm 15,16$ & $04,49 \pm 01,70$ \\
\hline & GLCM & $15 \times 15$ & $91,88 \pm 03,81$ & $91,88 \pm 03,81$ & $00,12 \pm 00,04$ & $11,45 \pm 01,29$ \\
\hline \multirow{4}{*}{ 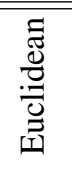 } & $\overline{\mathrm{ADH}}$ & $15 \times 15$ & 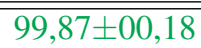 & 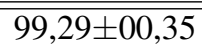 & $995,08 \pm 01,83$ & $04,07 \pm 00,73$ \\
\hline & MomSta & $15 \times 15$ & $99,86 \pm 00,22$ & $99,38 \pm 00,35$ & $95,95 \pm 01,62$ & $03,96 \pm 00,70$ \\
\hline & $\mathrm{HU}$ & $15 \times 15$ & $99,86 \pm 00,19$ & $99,37 \pm 00,37$ & $95,89 \pm 01,67$ & $03,99 \pm 00,72$ \\
\hline & GLCM & $15 \times 15$ & $99,84 \pm 00,21$ & $99,31 \pm 00,37$ & $95,30 \pm 02,05$ & $04,09 \pm 00,94$ \\
\hline \multirow{4}{*}{ 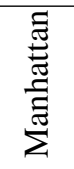 } & $\overline{\mathrm{ADH}}$ & $15 \times 15$ & $9 \overline{99,89 \pm 00,17}$ & $9 \overline{99,30 \pm 00,35}$ & $9 \overline{95,12 \pm 01,89}$ & $=04,07 \pm 00,75$ \\
\hline & MomSta & $15 \times 15$ & $99,86 \pm 00,22$ & $99,37 \pm 00,33$ & $95,94 \pm 01,61$ & $03,93 \pm 00,69$ \\
\hline & HU & $15 \times 15$ & $99,89 \pm 00,16$ & $99,36 \pm 00,38$ & $95,86 \pm 01,61$ & $04,04 \pm 00,81$ \\
\hline & GLCM & $15 \times 15$ & $99,86 \pm 00,20$ & $99,31 \pm 00,38$ & $95,32 \pm 01,94$ & $04,05 \pm 00,90$ \\
\hline
\end{tabular}

características e dimensões de máscaras utilizadas para a análise de vizinhança. Desta etapa, considerou-se os melhores resultados obtidos sob o ponto de vista das métricas de avaliação em relação ao padrão-ouro. Já a segunda fase consiste no comparativo de outros métodos da literatura baseados em MCA com novas energias externas como o GVF, VFC, THR mod e THR multi, mas também aqueles que propões novas energias internas como é o caso do CRISP e CRAD, além do sistema de segmentação SISDEP. Por fim, um comparativo é feito entre os resultados de cada fase. Com o objetivo de analisar o desempenho dos métodos, quatro métricas de avaliação estatística são empregadas neste trabalho: sensibilidade (Se), acurácia (Ac), além do coeficiente da correlação de Matthews (MCC) e distância de Hausdorff (HD).

\subsection{Análise dos resultados}

Os testes foram realizados em 72 imagens de pulmão distribuídas igualmente entre amostras com fibrose, DPOC e sadias. A primeira fase da análise considerou o desempenho do MCA OPS quando associado ao classificador OPF com diferentes distâncias para o treinamento das classes, nesta análise foram consideradas as distâncias Gaussiana, Euclideana e Manhattan. Para cada configuração foi executada uma segmentação utilizando os extratores de características ADH, MomSta, HU e GLCM, para cada extrator foram analisadas máscaras com dimensões de 3 à 15x15 pixels, com passo de 2 pixels.

Os resultados foram submetidos a uma avaliação quantitativa considerando as medidas Se, Ac e MCC, além destas, também foi avaliado o grau de proximidade entre a região segmentada pelo método e o padrão-ouro fornecido pelo especialista, através da métrica HD. Para analisar a vizinhaça com o ponto explorador, a máscara que apresentou melhores resultados foi com a dimensão $15 \times 15$ pixels para todas as combinações de extratores e classificadores OPF. A única exceção foi no classificador OPS com distância Gaussiana e extrator HU, onde a janela de melhor desempenho foi a 7x7, como pode ser visto na Tabela 1.

Considerando a acurácia, a proporção de predições corretas apresentou resultados superiores a 99\% para OPS com distância Euclidiana e Manhattan, para estas combinações o melhor resultado alcançado foi para o extrator MomSta com $99,38 \pm 00,35 \%$ e $99,37 \pm 00,33 \%$ respectivamente. Todavia, considerando o desvio 


\begin{tabular}{l}
$\begin{array}{l}\text { Tabela 2. Resultado das métricas de avaliação para o MCA OPS e demais } \\
\text { métodos considerados neste análise. }\end{array}$ \\
\cline { 2 - 5 } \\
\begin{tabular}{ccccc} 
MCA & Se & Ac & MCC & HD \\
\hline OPS & $99,88 \pm 00,20$ & $99,25 \pm 00,33$ & $95,05 \pm 01,62$ & $04,02 \pm 00,75$ \\
SISDEP & $99,86 \pm 00,19$ & $99,49 \pm 00,22$ & $96,25 \pm 01,65$ & $04,03 \pm 00,93$ \\
CRISP & $99,85 \pm 00,36$ & $99,02 \pm 00,54$ & $93,24 \pm 02,85$ & $04,59 \pm 01,42$ \\
CRAD & $99,69 \pm 00,33$ & $99,54 \pm 00,27$ & $96,42 \pm 03,09$ & $03,77 \pm 01,02$ \\
VFC & $99,62 \pm 00,48$ & $99,00 \pm 00,49$ & $92,92 \pm 03,20$ & $04,95 \pm 01,43$ \\
GVF & $99,58 \pm 00,93$ & $98,85 \pm 00,84$ & $91,41 \pm 07,89$ & $05,21 \pm 01,44$ \\
THR mod & $99,42 \pm 00,74$ & $98,81 \pm 00,75$ & $92,04 \pm 03,61$ & $04,98 \pm 01,54$ \\
THR multi & $98,75 \pm 01,54$ & $98,07 \pm 01,96$ & $88,27 \pm 07,97$ & $05,68 \pm 02,02$ \\
\hline
\end{tabular}
\end{tabular}

padrão apresentado, o extrator ADH apresentou resultados bastante similares para as mesmas distâncias. Nota-se, entretando, que MonSta não apresentou melhores resultados quando aplicado ao OPS com distância Gaussiana, sendo portanto superado pelo extrator $\mathrm{ADH}$, com valores de acurácia na ordem de 99,25 $\pm 00,33 \%$.

Já em relação a sensibilidade do método, os melhores resultados obtidos com as diferentes combinações do classificador OPF foram com o extrator ADH com sensibilidade $99,88 \pm 00,20 \%$ para a distância Gaussiana, 99,87 $\pm 00,18 \%$ para Euclidiana e, por fim, 99,89 $\pm 00,17 \%$ para Manhattan. O coeficiente MCC, corrobora com os resultados obtidos para a sensibilidade, como pode ser observado na Tabela 1, dentre as combinações do OPF com os demais extratores, o extrator ADH obteve os melhores resultados, considerando a distância Gaussiana, com valores proporcionais a 95,05 $\pm 01,62 \%$, superando os demais.

Conforme o grau de similaridade entre a região segmentada pelo OPS e o padrãoouro, considerando a medida de distância HD, os resultados na Tabela 1 mostram que o extrator MomSta apresentou melhores resultados com distâncias Euclidiana, com $03,96 \pm 00,70 \%$, e Manhattan com $03,93 \pm 00,69 \%$, apresentando porém resuldados insatisfatórios para a distância Gaussiana, sendo novamente superado pelo extrator ADH, com valor $04,02 \pm 00,75 \%$ em contrate com o valor $04,73 \pm 01,91 \%$ do MomSta, ressaltando assim a proximidade da área segmentada com o padrão de análise.

Diante dos resultados expostos na primeira etapa de análise, é possível concluir, portanto, que os melhores resultados para o OPS, com as diferentes configurações do OPF, foram obtidas pelos extratores MomSta e ADH, apresentando resultados bastante similares nas diferentes distâncias analisadas. Sendo que os melhores resultados considerando a distância Gaussiana foram obtidos pelo extrator $\mathrm{ADH}$, superando os demais.

Os resultados da segunda fase, descritos na Tabela 2, indicam que o índice $S e$ apresentou os melhores resultados para o OPS, sendo superior aos demais métodos com o melhor resultado sendo 99,88 $\pm 00,20 \%$. Já em relação ao índice de acurácia, os resultados apontam que o OPS é superior aos métodos VFC, GVF, HR mod, THR multi e CRISP, alcançando um índice de $99,25 \pm 00,33 \%$, e com resultado semelhante ao método CRAD. Acredita-se que estes resultados decorrem da característica adaptativa das energias internas do OPS, onde cada ponto explorador é apoiado pelo conhecimento fornecido pela classificação do OPF, identificando assim as bordas do objeto desejado, não sendo afetado, por exemplo, pelas estruturas internas ao parênquima pulmonar, proporcionando assim medidas qualitativas aceitáveis. 
Em relação ao índice de similaridade HD, o valor alcançados pelo método OPS foi da ordem de 04,02 $\pm 00,75 \%$, mostrando ser superior aos métodos CRISP, VFC, GVF, THR mod e THR multi. Para esta medida, o melhor resultado foi alcançado pelo método CRAD com valor $03,77 \pm 01,02 \%$, porém o alto desvio padrão pode representar uma instabilidade do método na segmentação, tornando seu resultado semelhante àqueles obtidos pelo SISDEP e OPS. Por fim, nas Figuras de 4(a) até (h) é ilustrada a diferença, em vermelho, do resultado de cada método comparado com o padrão-ouro, ressaltando a discrepância da segmentação com o padrão para imagens classificadas com DPOC.

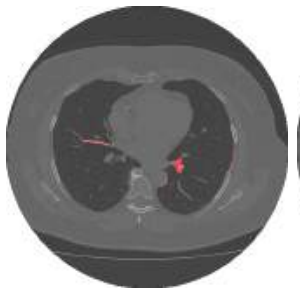

(a)

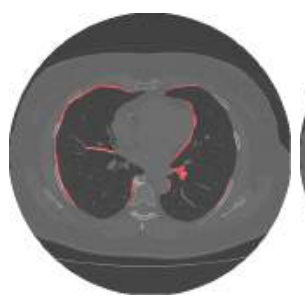

(e)

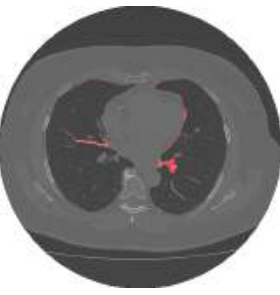

(b)

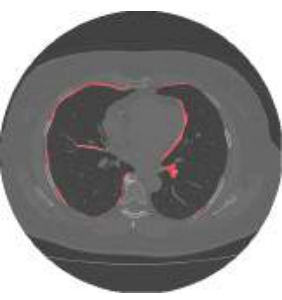

(f)

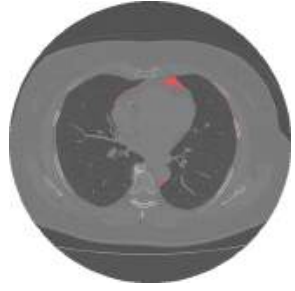

(c)

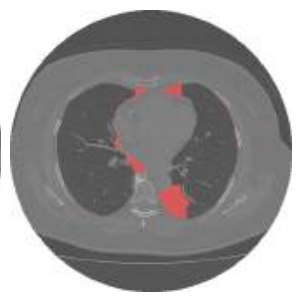

$(\mathrm{g})$

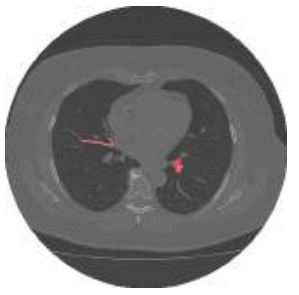

(d)

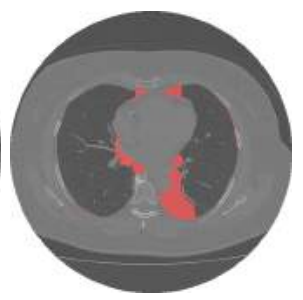

(h)

Figura 4. A diferença, na região em destaque, entre a segmentação do especialista e os resultados de segmentação via: (a) OPS, (b) SISDEP, (c) CRISP, (d) CRAD, (e) VFC, (f) GVF, (g) THR mod e (h) THR multi.

\section{Conclusões}

Os resultados obtidos neste trabalho, conforme Tabelas 1 e 2, mostram que o método de segmentação OPS apresenta resultados bastante promissores considerando seu potencial frente aos métodos baseados em contornos ativos já disponíveis na literatura. Alcançando valores aceitáveis de Acurácia e Sensibilidade, na ordem de 99,88\% $\pm 00,20$ e $99,25 \% \pm 00,33$, respectivamente. Expressando também índices de similaridade próximos ao padrão ouro fornecido pelo especialista, onde a distância HD sugere um grau de similaridade aceitável, semelhante ou superior aos demais métodos comparados.

Além disso, o extrator ADH apresentou índices superiores aos momentos estatíticos, invariantes, bem como ao extrator GLCM, considerando o OPF com distância Gaussiana. Isso mostra que o extrator têm potencial competitivo frente as demais técnicas, sugerindo a sua aplicabilidade como descritor de características em imagens de TC do tórax para a segmentação da região pulmonar. Com índices de similaridade ao padrãoouro superiores a 95\%, em média, podemos concluir que a abordagem proposta pode ser utilizada para auxílio ao diagnóstico médico em pneumologia.

\section{Referências}

S Avinash, K Manjunath, and S Senthil Kumar. An improved image processing analysis for the detection of lung cancer using gabor filters and watershed segmentation tech- 
nique. In International Conference on Inventive Computation Technologies (ICICT), volume 3, pages 1-6. IEEE, 2016.

Antonio Carlos da Silva Barros. Optimum Path Snakes: Novo Método de Contornos Ativos Adaptativo e Não-Paramétrico. PhD thesis, Universidade de Fortaleza, Fortaleza, CE, 2016.

John Hebert da Silva Felix, Paulo C. Cortez, Rodrigo C. S. Costa, Simone C. B. Fortaleza, Eanes D. B. Pereira, and Marcelo A. Holanda. Avaliação computacional de enfisema pulmonar em TC: comparação entre um sistema desenvolvido localmente e um sistema de uso livre. J. bras. pneumol, 35(9):868-876, 2009.

John Hebert da Silva Felix, Paulo C. Cortez, Pedro P. Rebouças Filho, TS Cavalcante, and Marcelo A. Holanda. Novo método de contornos ativo baseado na Transforma de Hilbert Radial. In V Latin American Congress on Biomedical Engineering CLAIB 2011 May 16-21, 2011, Habana, Cuba, pages 1051-1053. Springer, 2013.

Robert M Haralick, Karthikeyan Shanmugam, et al. Textural features for image classification. IEEE Transactions on systems, man, and cybernetics, 3(6):610-621, 1973.

Ming-Kuei Hu. Visual pattern recognition by moment invariants. IRE transactions on information theory, 8(2):179-187, 1962.

Michael Kass, Andrew Witkin, and Demetri Terzopoulos. Snakes: Active contour models. In International Journal of Computer Vision, volume 1, pages 321-331, 1987.

Bing Li and Scott T Acton. Vector field convolution for image segmentation using snakes. In 2006 IEEE International Conference on Image Processing, pages 1637-1640. IEEE, 2006.

Blair Mackiewich. Intracranial boundary detection and radio frequency correction in magnetic resonance images. PhD thesis, CiteSeer, 1995.

Aldisio Gonçalves Medeiros and Daniel S. Ferreira. Detecção de agrupamentos de microcalcificações em imagens digitais de mamografias. XV WIM - Workshop de Informática Médica, Recife - PE, 2015.

João Paulo Papa, Silas Evandro Nachif Fernandes, and Alexandre Xavier Falcão. Optimum-path forest based on k-connectivity: Theory and applications. Pattern Recognition Letters, 87:117-126, 2017.

Romain A. Pauwels, A. Sonia Buist, Peter M.A. Calverley, Christine R. Jenkins, and Suzanne S. Hurd. Global strategy for the diagnosis, management, and prevention of chronic obstructive pulmonary disease. American journal of respiratory and critical care medicine, 2012.

Pedro Pedrosa Rebouças Filho, Paulo C. Cortez, and Marcelo A. Holanda. Modelo de contorno ativo crisp: nova técnica de segmentação dos pulmões em imagens de TC. Revista Brasileira de Engenharia Biomédica, 27(4):259-72, 2011.

Pedro Pedrosa Rebouças Filho, Paulo C. Cortez, John Hebert Félix da Silva, Tarique da Silveira, and Marcelo A. H. Cavalcante. Modelo de contorno ativo crisp adaptativo 2D aplicado na segmentação dos pulmões em imagens de TC do tórax de voluntários sadios e pacientes com enfisema pulmonar. Revista Brasileira de Engenharia Biomédica, 29(4):363-376, 2013.

Pedro Pedrosa Rebouças Filho, Paulo C. Cortez, Antônio C. S. Barros, and Victor H. C. De Albuquerque. Novel adaptive balloon active contour method based on internal force for image segmentation-A systematic evaluation on synthetic and real images. Expert Systems with Applications, 41(17):7707-7721, 2014.

Chenyang Xu and Jerry L Prince. Snakes, shapes, and gradient vector flow. IEEE Transactions on image processing, 7(3):359-369, 1998. 